\title{
UN MODELO DE PERFILACIÓN PARA AGRESORES SEXUALES DESCONOCIDOS QUE AGREDEN EN LAS ENTRADAS DE LOS EDIFICIOS
}

\author{
Heriberto Janosch González ${ }^{1}$ Francisco Pérez Fernández², \\ Juan Enrique Soto Castro ${ }^{3}$
}

(1) Universidad Camilo José Cela, Madrid. Email: heribertojanosch@gmail.com.

(2) Facultad de Ciencias de la Salud. Universidad Camilo José Cela, Madrid.

Email: fperez@ucjc.edu.

${ }^{(3)}$ División de Formación y Perfeccionamiento. Policía Nacional.

Email: jesoto@cop.es.

Title: A profiling model for unknown sexual offenders that assault in the entrance of buildings.

Resumen: En el presente estudio pretendimos analizar las características de los autores de agresiones sexuales cuyo modus operandi es el de atacar a la víctima en portales o entradas de garaje de edificios. Nuestro estudio estuvo orientado a determinar si es posible predecir tales características a partir de los datos de la escena del crimen, analizando casos resueltos. Partiendo del contenido de sentencias judiciales condenatorias dictadas en España, y aplicando pruebas estadísticas de chi-cuadrado, odds-ratio, y regresión logística binaria, hemos obtenido resultados alentadores, en el sentido de que algunas de las variables de la escena del crimen permiten predecir algunas características de los autores. Dado que las variables de la escena del crimen utilizadas aquí son conocidas por el equipo policial al inicio de la investigación, la predicción de características del agresor sexual podría ser de utilidad a la hora de priorizar sospechosos.

Palabras clave: Agresión sexual - Características del agresor Escena del crimen - España 
Abstract: This paper aims at analysing the features of the perpetrators of sexual assaults whose modus operandi consists of assaulting the victim in doorways or entrances of garage and buildings. Its objective was to determine whether it is possible to predict these features moving from the information regarding the crime scene, by means of analysing solved cases. Moving from the content of convicting judgments issued in Spain, and by applying statistical tests of chi-square, odds-ratio, and binary logistic regression, we have obtained encouraging results, in that some variables in the crime scene allow to predict some features of these offenders. Since the variables of the crime scene that have been used here are known to the police team at the beginning of the investigation, prediction of the sexual offender's features could be useful to prioritize suspects.

Keywords: Sexual assault - offender's features - crime scene - Spain

\section{Introducción}

Las agresiones sexuales cometidas por individuos desconocidos para las víctimas suponen un problema criminológico en España. Implican un gran sufrimiento para las víctimas, en su gran mayoría mujeres, al mismo tiempo que, en algunos casos, provocan una gran alarma social. Según el Informe sobre Delitos contra la Libertad e Indemnidad Sexual elaborado en 2018 por la Secretaría de Estado de Seguridad del Ministerio del Interior, se produjeron ese año casi catorce mil delitos de índole sexual (2018). De ese total, más de 5.500 se produjeron en viviendas y en anexos. Una de las modalidades frecuentes de estos crímenes es aquella en la que el agresor sexual, por lo general un hombre actuando en solitario, ataca a una mujer en la entrada de un edificio, habitualmente de viviendas, en el portal de acceso, o a veces en la entrada del garaje. A estos agresores sexuales se les conoce como "portaleros» en el argot policial. El esclarecimiento de agresiones sexuales cometidas por individuos desconocidos para las víctimas conlleva mayor dificultad que aquellas en las que existe algún tipo de vínculo entre víctima y victimario que permite la identificación de éste y la obtención, en su caso, de indicios que puedan convertirse en medios de prueba (Corovic, Christianson y Bergman, 2012). A las Fuerzas y Cuerpos de Seguridad del Estado se les plantea el imperativo de individualizar a estos delincuentes de víctima desconocida, para ponerlos a disposición judicial, y de esa forma evitar que continúen cometiendo esta clase de delitos. Tengamos en cuenta que la psicología de estos individuos funciona habitualmente mediante un proceso de escalada, lo cual significa que, en relación a su potencial peligrosidad, cometerán más de una agresión sexual en el transcurso de su carrera delictiva (Pueyo y Redondo, 2007).

Entre las numerosas técnicas que están a disposición de los investigadores policiales se encuentra la de la perfilación criminológica, 
coloquialmente "perfilación», "perfilado» o "profiling», que puede resultar de utilidad para priorizar sospechosos, cuando no hay otro tipo de evidencias, como muestras de ADN del agresor, algún testigo que le identifique, o una confesión (Canter y Youngs, 2009; Douglas et al., 1986; Soto Castro, 2017; Sotoca, González y Halty, 2019; Turvey, 2012). La técnica de la perfilación se basa en inferir, a partir de datos de la escena del crimen, de las características de la víctima, de sus declaraciones y las de posibles testigos, del contenido de los informes forenses, del entorno físico y social, de la reconstrucción del hecho, y otras informaciones disponibles, las posibles características psicológicas, criminológicas y sociodemográficas del autor. Puede verse que la definición es muy compleja, pero suele resumirse, en línea con las propuestas de la denominada Psicología Investigativa, con las expresiones $" \mathrm{~A}=>\mathrm{C}$ », «Acciones $=>$ Características» (Canter y Youngs, 2009), o también «Escena del Crimen => Características del Autor». Utilizaremos ésta última expresión en el presente artículo. Con esta técnica no podríamos individualizar al delincuente hasta el punto de lograr una identidad única, pero podríamos obtener un perfil hipotético de la clase de persona que cometió el delito que restringe el número de posibles sospechosos, lo que redunda en una mejor y más eficiente investigación policial.

Los principios fundamentales de la perfilación son las hipótesis de consistencia, homología y diferenciación. La consistencia se refiere a que los delincuentes suelen cometer crímenes similares a lo largo del tiempo, siguiendo en lo posible el mismo modus operandi, reforzado por el hecho de que, mientras no son detectados, sus actos les han dado buenos resultados en el pasado. Por lo tanto, las escenas de los crímenes de un delincuente en particular serán similares entre sí. La hipótesis de la homología afirma que, si dos escenas de crímenes cometidos por dos individuos son similares entre sí, entonces las características de estos dos autores serán también similares entre sí. Por último, la diferenciación es la contrapartida de la homología, que supone que, si dos escenas de crímenes cometidos por dos autores son diferentes entre sí, entonces las características de ambos individuos también serán diferentes. Si el principio de la homología se comprueba, los otros dos también serán necesariamente válidos, a partir de deducciones o inferencias lógicas, por lo que es importante saber si se han encontrado evidencias de la validez de la homología. En tal sentido la literatura arroja resultados que han sido contrapuestos e inconcluyentes. Mientras que en algunos estudios no se ha podido comprobar el supuesto de la homología (por ejemplo, Mokros y Alison, 2002), en otros sí se ha logrado (por ejemplo, Goodwill et al., 2016). Ello implica que aún es necesaria más investigación en relación a este tópico, así como un perfeccionamiento metodológico de los procedimientos que redunden en resultados confiables (Snook et al., 2007).

Existen dos enfoques en la práctica de la perfilación, la deductiva y la inductiva. La deductiva (Turvey, 2012) pretende realizar el perfil del delincuente analizando exclusivamente el hecho delictivo puntual, dedu- 
ciendo a partir de la escena del crimen las características del autor. La inductiva (Canter y Youngs, 2009), por su parte, intenta basar el perfilado en la información suministrada por bases de datos de delitos similares, resueltos, donde no solo aparecen los detalles de escenas de crímenes similares, sino también las características de los autores de tales delitos. En la práctica, se utilizan ambos enfoques, y se recurre a la creación de hipótesis, recurriendo al procedimiento que Charles Peirce denominaba abducción (Soto Castro, 2017), sobre las características del autor. Tratándose cualquier perfilación en particular de un sistema de problemas inversos (Janosch González, 2020), lo ideal es recurrir a todas las herramientas válidas que se tenga a disposición, y de esa forma ayudar en lo posible al equipo de investigación policial a esclarecer el delito. En todo caso, ha de tenerse presente que el perfil debe ser adecuadamente presentado a los investigadores del caso y, asimismo, ha de permanecer sujeto a constante revisión en función de las ulteriores evidencias que el trabajo policial arroje (Turvey, 2012).

No hemos encontrado entre los estudios previos ninguno que trate específicamente de esta clase de delitos sexuales cometidos en portales de edificios, por lo que pensamos que el presente podría ser el primero de este tipo. Sin embargo, hay numerosos estudios sobre agresiones sexuales de autores desconocidos para las víctimas en general, algunos de los cuales, se refieren de manera específica a su relación con la perfilación (véase por ejemplo: Alison y Stein, 2001; Almond y sus colaboradores, 2018; Canter et al., 2003; Canter y Heritage, 1990; Häkkänen, Lindlöf y Santtila, 2004; Hewitt, Chopin y Beauregard, 2020; Lehmann et al., 2013). Aunque la finalidad de estos estudios es la de vincular características de las diferentes escenas de crímenes con las diferentes características de los autores, se utilizan en el análisis diversos enfoques metodológicos.

Así, por ejemplo, Häkkänen, Lindlöf y Santtila (2004) analizan en primer lugar las características de las escenas de 100 crímenes de violación por desconocidos que han sido esclarecidos, estableciendo tres temáticas mediante un procedimiento estadístico de escalado multidimensional, que podrían traducirse como Hostilidad, Robo y (falsa) Intimidad. En la temática de Hostilidad se registraron conductas del agresor tales como amenaza de muerte, violencia, felación o penetración vaginal por detrás; en la de Robo, además del robo propiamente dicho, se registraron conductas de penetración anal o amordazamiento de la víctima; y en la de (falsa) Intimidad, besos, cumplidos, masturbación, y deseos de entablar conversación con la víctima, preguntando el agresor sobre aspectos de la vida de la misma, o revelando él mismo aspectos de su propia historia de vida. En segundo lugar, procedieron a analizar, también mediante escalado multidimensional, las características de los agresores sexuales, obteniendo cuatro agrupaciones: Psiquiátrico/Mayor, Criminal orientado a la propiedad, Criminal orientado a la violencia, y Convencional. Finalmente trataron de determinar si estas cuatro agrupaciones de características de 
autores correlacionaban significativamente con las tres temáticas de la escena del crimen. Hallaron una correlación significativa entre una escena del crimen de tipo Robo y unas características del delincuente como Criminal orientado a la propiedad.

Hewitt, Chopin y Beauregard (2020), por su parte, analizan las distancias euclídeas entre el hogar de la víctima y el del agresor sexual, entre el hogar de la víctima y el del asalto sexual, y entre el hogar del agresor sexual y el del asalto sexual, e intentan predecirlas a partir de variables correspondientes a la escena del crimen tales como factores temporales (de día, durante el fin de semana, en el verano, etcétera), características ambientales (residencial, comercial, parking, etcétera), actividad de la víctima justo antes de la agresión (actividades domésticas, socializando, prostituyéndose, etcétera), y tipo de motivación del agresor sexual. En este último aspecto parece haber consenso en que los agresores sexuales desconocidos para la víctima actúan con una o más de las siguientes motivaciones diferentes: oportunista; compensatorio; sádico; poder y control; y enfadado (Hewitt, Chopin y Beauregard, 2020). La muestra, importante, consistía en 1.009 casos de violaciones en Francia. Entre otras pruebas, aplicaron análisis bivariados y multivariados. Analizaron las variables dependientes (características del autor, en este caso las distancias) en un análisis bivariado con cada una de las variables independientes (de la escena del crimen), y con aquellas variables que producían correlaciones significativas practicaron un análisis de regresión logística negativa binomial secuencial para cada variable dependiente. Entre los múltiples resultados podemos citar, a modo de ejemplo, los siguientes: los violadores con motivación compensatoria atacan a sus víctimas más cerca de la residencia de las mismas que aquellos violadores que muestran otras motivaciones; los violadores con motivación oportunista son los que más cerca viven de sus víctimas; y los violadores con motivación mixta de enfado/poder y control son los que más lejos viven de sus víctimas.

En un último ejemplo, el del estudio realizado por Almond y sus colaboradores (2018), la metodología utilizada fue similar. Los autores estudiaron las características del delincuente referidas a sus antecedentes criminales por diferentes hechos delictivos, tales como robo, drogas, o violencia, a partir de una muestra de 210 agresores sexuales británicos. Establecieron correlaciones entre cada una de las variables referentes a la escena del crimen con cada una de las variables referentes a las características del autor, y descartaron las correlaciones no significativas. Con el resto procedieron a realizar análisis multivariados mediante la aplicación de pruebas de regresión logística binaria, una prueba para cada variable dependiente. Entre los resultados podemos citar, a modo de ejemplo, los siguientes: la probabilidad de que el agresor sexual tenga antecedentes criminales crece si en el acto de la agresión sexual le robó algún valor a la víctima y forzó la entrada en la vivienda; la probabilidad de que el agresor sexual tenga antecedentes por robo crece si en el acto de la agresión 
sexual forzó la entrada de la vivienda e hizo referencias a la policía; y la probabilidad de que el agresor sexual tenga antecedentes por drogas crece si en el acto de la agresión sexual hizo referencia a la policía.

En el presente estudio pretendemos analizar las características de los autores de agresiones sexuales cuyo modus operandi es el de atacar a la víctima en portales o entradas de garaje de edificios, generalmente de viviendas. Nos centraremos en aquellos agresores sexuales hombres, que actúan en solitario, y cuyas víctimas son mujeres que en el momento de la agresión tenían 13 o más años, siendo el agresor desconocido para la víctima, hasta un máximo de 24 horas antes de la comisión del delito. En cuanto a la escena del crimen, focalizaremos en los datos que suelen estar a disposición del equipo de investigación policial cuando todavía no se individualizó al autor del delito, y en cuanto a las características del agresor, nos centraremos en aquellas que poseen un valor operativo para la investigación policial.

\section{Método}

La muestra del presente estudio ha sido tomada de la base de datos del Centro de Documentación Judicial (CENDOJ) del Consejo General del Poder Judicial (CGPJ). Se han tomado solo las sentencias judiciales condenatorias, dictadas entre 2015 y 2019, que describen 57 agresiones sexuales, cometidas por 39 hombres desconocidos para las víctimas, todas mujeres de 13 o más años de edad. Consideramos una edad mínima de 13 años para la víctima porque, como bien expresan Pérez Ramírez y colaboradores (2018), las víctimas menores de 13 años son consideradas prepúberes, y estarían en la categoría delictiva del abuso sexual de niñas. Los agresores atacaron a sus víctimas, actuando en solitario, en portales, en entradas de edificios o en entradas de garajes.

Un análisis de contenido de las sentencias nos permitió detectar y seleccionar un conjunto de 19 variables, tanto referidas a las características del agresor sexual como a la escena del crimen (ver tabla 1). Las 19 variables son nominales dicotómicas, siendo sus posibles valores 0 (ausencia) o 1 (presencia).

\begin{tabular}{|l|l|}
\hline \multicolumn{2}{|c|}{ TABLA 1: VARIABLES RESULTANTES DEL ANÁLISIS DE CONTENIDO } \\
& \multicolumn{1}{c|}{ DE LAS SENTENCIAS } \\
\hline \multicolumn{2}{|c|}{} \\
\hline Consumo & El agresor consumió alcohol o drogas antes de cometer el delito \\
\hline Drogas & El agresor consumió drogas antes de cometer el delito \\
\hline Alcohol & El agresor consumió alcohol antes de cometer el delito \\
\hline
\end{tabular}

(C) UNED. Revista de Derecho Penal y Criminología, 3. ${ }^{a}$ Época, n. ${ }^{\circ} 24$ (2020) 


\begin{tabular}{|l|l|}
\hline \multicolumn{2}{|c|}{ TABLA 1: VARIABLES RESULTANTES DEL ANÁLISIS DE CONTENIDO } \\
DE LAS SENTENCIAS \\
\hline Cvi_disminuidas & $\begin{array}{l}\text { El agresor tenía las capacidades volitivas e intelectivas disminuidas } \\
\text { durante la comisión del delito }\end{array}$ \\
\hline Psiquiatrico & El agresor padece problemas psiquiátricos \\
\hline Ag_serie & El agresor es serial \\
\hline Antecedentes & El agresor tiene un historial de antecedentes judiciales en general \\
\hline Ant_L_sexual & $\begin{array}{l}\text { El agresor tiene un historial de antecedentes judiciales por delito } \\
\text { sexual }\end{array}$ \\
\hline Edad_D & El agresor tenía 30 años o más en el momento de la agresión \\
\hline & Variables referentes a la escena del crimen \\
\hline Resistencia & La víctima ofreció resistencia \\
\hline Terceros & Aparecieron terceras personas durante la agresión \\
\hline Interrumpida & La agresión se interrumpió en algún momento \\
\hline Oscuridad & La agresión ocurrió en horas de oscuridad \\
\hline Arma & El agresor utilizó un arma \\
\hline Penetracion & Hubo penetración vaginal, oral, y/o anal \\
\hline Hay_robo & El agresor robó objetos de valor a la víctima \\
\hline Disfraz & El agresor utilizó algún tipo de disfraz \\
\hline Finde_D & La agresión se produjo durante un fin de semana \\
\hline Edad_vict_D & La víctima tenía 20 años o más en el momento de la agresión \\
\hline
\end{tabular}

En cuanto a la variable Oscuridad se procedió de la siguiente manera: si la agresión sexual ocurrió circunstancialmente entre las horas de la puesta del Sol y su siguiente salida, se le asignó el valor 1. En el caso contrario se le asignó el valor 0. Por otro lado, a la variable Finde_D se le asignó un valor 1 si la agresión sexual sucedió entre el mediodía del viernes y el mediodía del siguiente lunes. En el caso contrario se le asignó el valor 0 .

Para los análisis estadísticos hemos seguido la metodología empleada en el estudio de Almond y sus colaboradores (2018). Esta metodología resulta ser la más adecuada, según el estudio de Goodwill, Alison y Beech (2009), en el que se compararon los modelos tipológicos, temáticos y multivariados. El objetivo del presente estudio fue predecir variables dicotómicas referidas a las características del autor en función de la escena del crimen tal y como se describe en la información judicial. El análisis de datos se realizó en dos fases. En la fase 1, se utilizaron análisis de chi-cuadrado para examinar si había asociaciones significativas entre las variables de la escena del crimen y las variables referidas a las características del autor. 
También se calcularon las razones de probabilidad (odds-ratio) de cualquier asociación significativa, para indicar la probabilidad estadística de que un delincuente tenga cierta característica basada en su comportamiento en la escena del crimen (Goodwill, Alison y Beech, 2009). Siguiendo a Chen, Cohen y Chen (2010) se consideró que las razones de probabilidad (odds-ratio) significaban tamaños de efecto pequeños $(<1,5)$, medianos $(1,5-5)$ o grandes $(>5)$. Por último, siempre en la fase 1, se indagó en las posibles asociaciones entre las variables de la escena del crimen que tenían una asociación significativa con variables de características del autor. Para la fase 2 , cualquier variable de la escena del crimen, que identificada por los análisis de chi-cuadrado se asociaba significativamente a alguna variable de las características del delincuente, se ingresó en los análisis de regresión logística. De esta manera se garantizó que las variables del delito produjeran el modelo predictivo óptimo para las variables de características del autor. Estos modelos de regresión logística evalúan la capacidad predictiva de un conjunto de variables independientes (de la escena del crimen) sobre una variable dependiente categórica (característica del autor), indicando la contribución de cada variable predictora al modelo que se propone. Las pruebas estadísticamente significativas indican que la variable contribuye a la precisión predictiva de la variable de resultado.

\section{Resultados}

La edad de los agresores sexuales al momento de cometer el delito se incluía en 48 de los 57 casos analizados, siendo la media de 30,10 años y la desviación estándar 7,36, con un mínimo de 18 y máximo de 47 . Por su parte, la edad de las víctimas en el momento del asalto sexual se incluía en 30 de los 57 casos estudiados, siendo la media de 23,67 años y la desviación estándar de 13,57, con un mínimo de 13 y un máximo de 83. Las edades fueron dicotomizadas según sus medianas para incluirlas en el estudio.

Los porcentajes válidos de presencia (valor 1) para cada variable dicotómica definida, tras el pertinente estudio de la documentación, se expresan en la Tabla 2.

\begin{tabular}{|l|l|}
\hline \multicolumn{2}{|c|}{ TABLA 2: PORCENTAJES VÁLIDOS PARA LA PRESENCIA DE VARIABLES } \\
DICOTÓMICAS
\end{tabular}

(C) UNED. Revista de Derecho Penal y Criminología, 3. ${ }^{a}$ Época, n. ${ }^{\circ} 24$ (2020) 


\begin{tabular}{|l|l|}
\hline \multicolumn{2}{|c|}{ TABLA 2: PORCENTAJES VÁLIDOS PARA LA PRESENCIA DE VARIABLES } \\
\hline DICOTÓMICAS
\end{tabular}

A continuación, se realizaron los análisis de chi-cuadrado para explorar asociaciones significativas entre comportamientos de la escena del crimen y las características del autor del delito. La Tabla 3 resume los resultados que muestran las razones de probabilidad (odds-ratio) entre los comportamientos de la escena del crimen de agresión sexual por un desconocido y las características del delincuente. No se incluyeron aquellas variables, tanto de la escena del crimen como de las características del autor, que no arrojaron ninguna asociación significativa con otras variables.

\begin{tabular}{|l|l|l|l|l|l|l|}
\hline \multicolumn{7}{|c|}{ TABLA 3: RAZONES DE PROBABILIDADES ENTRE CARACTERÍSTICAS } \\
DEL AUTOR Y ESCENA DEL CRIMEN \\
\hline & Consumo & Alcohol & $\begin{array}{l}\text { Cvi_ } \\
\text { disminuidas }\end{array}$ & Psiquiatrico & Ag_serie & Edad_D \\
\hline Resistencia & & & 15,05 & 14,59 & & \\
\hline Interrumpida & 7,75 & 7,75 & 5,43 & & & \\
\hline Oscuridad & & & & & & 5,36 \\
\hline
\end{tabular}


TABLA 3: RAZONES DE PROBABILIDADES ENTRE CARACTERÍSTICAS DEL AUTOR Y ESCENA DEL CRIMEN

\begin{tabular}{|l|l|l|l|l|l|l|}
\hline Arma & & & 0,10 & 0,10 & & \\
\hline Penetracion & 0,10 & 0,10 & 0,07 & 0,10 & & \\
\hline Finde_D & & & & & 0,14 & 6,30 \\
\hline
\end{tabular}

Resistencia. Encontramos que en las escenas del crimen en las que hubo resistencia de la víctima, fue 15 veces más probable que el autor del delito tuviera las capacidades volitivas e intelectivas disminuidas, $\chi^{2}$ (1) $=9,290, \mathrm{p}<0,01, \mathrm{y} 14$ veces más probable que el delincuente padeciera de un trastorno psiquiátrico, $\chi^{2}(1)=6,035, \mathrm{p}<0,05$.

Interrumpida. En las escenas del crimen donde la agresión sexual fue interrumpida por cualquier motivo, resultó 7 veces más probable que el autor del delito hubiera consumido alcohol o drogas, $\chi 2(1)=4,244$, $\mathrm{p}<0,05 ; 7$ veces más probable que el autor hubiera consumido alcohol, $\chi^{2}(1)=4,244, p<0,05$; y 5 veces más probable que el delincuente hubiera tenido las capacidades volitivas e intelectivas disminuidas, $\chi^{2}(1)=5,986$, $\mathrm{p}<0,05$.

Oscuridad. En los delitos que ocurrieron durante las horas nocturnas fue 5 veces más probable que el autor tuviera 30 o más años, $\chi 2(1)=$ 4,288, $\mathrm{p}<0,05$.

Arma. En las agresiones sexuales en las que el autor utilizó un arma, fue más probable que el delincuente no tuviera las capacidades volitivas e intelectivas disminuidas durante la comisión del hecho, $\chi 2(1)=5,874$, $\mathrm{p}<0,05$; y más probable que no padeciera un trastorno psiquiátrico, $\chi^{2}$ (1) $=4,215, p<0,05$. Nótese que en ambas correlaciones las asociaciones se dieron de manera inversa.

Penetración. En los delitos que hubo penetración vaginal, oral, y/o anal fue más probable que el autor no hubiera consumido drogas o alcohol, $\chi 2(1)=5,663, \mathrm{p}<0,05$; más probable también que no hubiera consumido alcohol, $\chi 2(1)=5,663, p<0,05$; más probable que no tuviera las capacidades volitivas e intelectivas disminuidas, $\chi^{2}(1)=12,836$, $\mathrm{p}<0,001 ; \mathrm{y}$, en la línea de lo precedente, más probable que no padeciera un trastorno psiquiátrico, $\chi 2(1)=5,663, p<0,05$. Nótese que en las cuatro correlaciones las asociaciones fueron inversas.

Fin de Semana. En los casos que el delito se cometió en fines de semana fue más probable que el agresor no fuera serial, $\chi 2(1)=6,898$, $\mathrm{p}<0,01$; y 6 veces más probable que el agresor tuviera 30 o más años, $\chi 2$ $(1)=7,743, p<0,01$. Nótese que en la primera correlación la asociación resultó ser inversa.

También se han determinado posibles asociaciones significativas entre las 6 variables de la escena del crimen descritas en la Tabla 
3, tomadas de a dos en dos. Un análisis de chi-cuadrado reveló las siguientes asociaciones significativas. Interrumpida y Penetracion, asociación inversa: $\chi^{2}(1)=16,245, \mathrm{p}<0,01$. Interrumpida y Resistencia, asociación directa: $\chi 2(1)=17,576, \mathrm{p}<0,01$. Penetracion y Resistencia, asociación inversa: $\chi 2(1)=16,352, \mathrm{p}<0,01$.

Por último, se debe significar que se hallaron dos modelos multivariados utilizando la regresión logística binaria.

Capacidades volitivas e intelectivas disminuidas. Encontramos que dos componentes de la escena del crimen estaban asociados con que el autor del delito haya actuado con sus capacidades disminuidas: la no utilización de un arma y que la agresión sexual se viera interrumpida. El modelo resultante fue significativo, $\chi^{2}(2)=12,241, p<0,01$. Ambas variables realizaron una contribución estadísticamente significativa al modelo. El modelo en su conjunto explicó entre el 19\% ( $\mathrm{R}^{2}$ de Cox y Snell) y el $30 \%$ ( $\mathrm{R}^{2}$ de Nagelkerke) de la varianza de las capacidades disminuidas y clasificó correctamente el 78,9\% de los casos.

Edad del autor. Encontramos que dos componentes de la escena del crimen estaban asociados con el rango de 30 o más años de edad del autor del delito: si éste actuó en el fin de semana y si la agresión sexual ocurrió durante la noche. El modelo resultante fue significativo, $\chi^{2}(2)=12,622, p<0,01$. Ambas variables realizaron una contribución estadísticamente significativa al modelo. El modelo en su conjunto explicó entre el 24\% ( $\mathrm{R}^{2}$ de Cox y Snell) y el 32\% ( $\mathrm{R}^{2}$ de Nagelkerke) de la varianza del rango de 30 o más años de edad y clasificó correctamente el $73,9 \%$ de los casos.

\section{Discusión y conclusiones}

Con una media de 30 años, el rango de edad de los agresores sexuales "portaleros» de nuestra muestra española es de 18 a 47 años. No hemos encontrado casos donde el agresor sea menor de 18 o mayor de 47. Por otro lado, con una media de 24 años, el rango de edades de las víctimas oscila entre los 13 y los 83 años. En este último caso el límite inferior lo hemos impuesto, y el límite superior es lo suficientemente grande como para pensar que la edad de la víctima no la protege de sufrir una agresión sexual en portales. Cabe pensar, en relación a este asunto, que la cuestión de la edad de la víctima seleccionada guarda relación con otros elementos asociados al propio agresor, o bien a las circunstancias del delito, tales como sus propias preferencias psicosexuales, o bien elementos aleatorios como la oportunidad, pero quizás por no disponer de suficientes datos acerca de la edad de la víctima, estas posibles relaciones no salieron a la luz. 
De acuerdo a los resultados de nuestra muestra el agresor consumió alcohol en 1 de cada 10 casos, y drogas solo en el 3,5\% de los mismos. Además, tenía sus capacidades volitivas e intelectivas disminuidas en 1 de cada 5 casos, y padecía problemas psiquiátricos en 1 de cada 10. Esto nos indica que, en la mayoría de los hechos, no hubo consumo excesivo de alcohol o drogas, ni el agresor padecía problemas psiquiátricos ni tenía sus capacidades disminuidas. Por lo tanto, cabe deducir la presencia de una intencionalidad consciente en la comisión de esta clase de delitos, aunque la planificación haya sido de tipo condicional, "si se da la oportunidad, lo haré de esta manera». También se ve una intencionalidad y premeditación en el hecho de que 8 de cada 10 casos ocurrieron en horario nocturno, cuando hay menos personas en la calle y hay más dificultad para observar el hecho por parte de extraños, así como una menor probabilidad de ser interrumpido. No obstante los cuidados que pareció haber puesto el delincuente, ocurrió que en uno de cada cuatro casos aparecieron testigos, o fuerzas policiales durante la agresión, y en la mitad de los casos la víctima ofreció alguna resistencia a los planes del agresor. En este sentido, destacamos que quizás el agresor no realiza su ataque donde quiere, sino donde puede, eligiendo, no obstante, aquellos lugares y momentos en los que considera que las posibilidades de cometer la agresión con éxito sean mayores. De ahí que sea mayor la casuística en horario nocturno y fundamentalmente contra víctimas que están solas, aunque pueda darse, por la configuración de la vía pública y del inmueble, la potencial aparición de testigos. Cabe deducir de todo lo expuesto, por lo demás, que en esta clase de agresiones sexuales cometidas por desconocidos existe un necesario componente de merodeo en busca de víctima, lugar y ocasión.

Nótese que, como preveíamos, en dos de cada tres hechos delictivos el agresor ya había cometido otro delito de agresión sexual, y en la cuarta parte de los casos tenía antecedentes judiciales por delito sexual. De ahí la necesidad que este tipo de crímenes no quede impune, y los agresores sean detenidos y puestos a disposición judicial, a fin de evitar que sigan agrediendo sexualmente a futuras víctimas y sean sometidos a los adecuados mecanismos de reinserción que tiendan a una reducción de su potencial peligrosidad. Sobre todo, teniendo en cuenta que en 4 de cada 10 casos han utilizado un arma (cuchillo o similar) para cometer el crimen, y que, asimismo, las posibilidades de que la víctima muestre resistencia son elevadas.

Es interesante observar que la agresión se interrumpió en algún momento en 4 de cada 10 casos, y además hubo penetración vaginal, oral, y/o anal en 6 de cada 10 casos. Cabe preguntarse, por tanto, si las agresiones sexuales que fueron interrumpidas no habrían terminado en penetración de llegar a término. Así parece, sobre todo si se tiene en cuenta que hay una asociación inversa significativa entre las variables Interrumpida y Penetracion. De los resultados de los test de chi-cuadrado y odds-ratio sabemos que, cuando la víctima ofreció resistencia, creció la 
probabilidad de que el delincuente tuviera las capacidades disminuidas durante el hecho, ya fuera por consumo de alcohol o drogas, o bien por trastorno psiquiátrico. Cabe suponer, pues, que ello pudo ser percibido por la víctima, y de ahí su intento, fallido o no, de ofrecer resistencia al ataque. Esto se relaciona con los casos donde la agresión fue efectivamente interrumpida, con respecto a los cuales los resultados muestran que crecía la probabilidad de que el agresor hubiera consumido alcohol o drogas, o que tuviera las capacidades volitivas e intelectivas disminuidas. También sabemos que el hecho de que la víctima ofrezca resistencia disminuye las posibilidades de que haya penetración, a la par que aumentan significativamente las probabilidades de que la agresión no finalice. No obstante, debemos ser cautos en relación a este asunto, pues hay evidencias en la literatura de que, si bien la resistencia de la víctima puede llevar a la interrupción de la agresión, en algunos casos la resistencia puede poner más violento al delincuente, especialmente en los casos de agresores de perfil colérico y/o sádico, causando incluso graves consecuencias físicas para la víctima, e incluso la muerte en casos extremos (Gibbens, Way y Soothill, 1977; Holmstrom y Burgess, 1980). Hay que considerar también que la penetración puede no producirse por otros factores, como que el agresor haya consumido drogas o alcohol, que padezca de un trastorno psiquiátrico, que presente algún tipo de disfunción sexual, aunque sea momentánea, o que, en definitiva, tenga sus capacidades volitivas y cognitivas disminuidas. Sin embargo, estas deducciones debieran en un futuro contrastarse con la información contenida en las evaluaciones periciales de los agresores, de las que no se ofrece información precisa en los informes judiciales.

La utilización de un arma correlaciona inversamente con la de tener las capacidades disminuidas y con el hecho de padecer un trastorno psiquiátrico, lo que sugiere que el agresor sexual utiliza el arma para amedrentar conscientemente a su víctima, y lograr así sus fines. Actuar bajo la protección de la oscuridad también se hace conscientemente, y de tal modo parecen hacerlo los delincuentes de 30 años o más, quienes tendrían más experiencia y habilidad para no ser detectados. Siempre en el caso de los "portaleros», los autores seriales, también con más experiencia, parecen actuar más en días laborables que los autores a los que solo se les ha contabilizado una agresión sexual. Quizás, cabe aventurar, esto sea debido simplemente a que cometen más delitos, y tienen más probabilidad de que los mismos se produzcan en días no festivos, o bien a que aprovechen circunstancias propicias, como las pausas o desplazamientos laborales, para la comisión de los ataques (Greenfeld, 1997). La relación de estos delitos con otro tipo de criminalidad concurrente se demuestra al tener en cuenta que en 3 de cada 10 casos los autores aprovecharon la agresión sexual para robar objetos de valor a las víctimas, o viceversa.

Los dos modelos de regresión logística amplían las posibilidades de predicción que pueden tener a su disposición los equipos policiales que 
investigan estos delitos. Si el agresor no utilizó un arma y la agresión fue interrumpida, es posible predecir que el autor tenía las capacidades volitivas o cognitivas disminuidas $\mathrm{y}$, por lo tanto, se deberá buscar a una persona de esas características: un adicto a las drogas o al alcohol, un enfermo psiquiátrico, o una persona con algún historial previo de trastornos psíquicos. En cuanto a los «portaleros» que actúan los fines de semana y aprovechándose de la oscuridad, podemos predecir que su edad será de 30 años en adelante.

Este estudio ha tenido ciertas limitaciones. En primer lugar, se ha basado en casos en los que el autor fue detenido. No sabemos si las características de aquellos delincuentes que han escapado, hasta ahora, a la acción de la justicia, podrían ser diferentes en algunos aspectos a los incluidos en nuestra muestra. En segundo lugar, hemos utilizado sentencias judiciales, documentos cuya finalidad no es la investigación científica y que, por tanto, carecen de detalles relevantes para la comprensión de la motivación de las agresiones sexuales. En tercer lugar, la muestra de 57 casos podría resultar insuficiente, y sería deseable que se ampliara en futuros estudios.

Los autores declararon no tener ningún conflicto potencial de intereses con respecto a la investigación, autoría o publicación de este artículo.

Los autores no recibieron apoyo financiero para la investigación, autoría o publicación de este artículo.

\section{Referencias bibliográficas:}

Alison, L. J., y Stein, K. (2001). Vicious circles: How accounts of stranger sexual assault reflect abusive variants of conventional interactions. The Journal of Forensic Psychiatry, 12, 515-538.

Almond, L., McManus, M., Bal, A., O’Brien, F., Rainbow, L., y WebB, M. (2018) Assisting the Investigation of Stranger Rapes: Predicting the Criminal Record of U.K. Stranger Rapists From Their Crime Scene Behaviors. J Interpers Violence.

Canter, D. V., Bennell, C., Alison, L. J., y Reddy, S. (2003). Differentiating sex offences: A behaviorally based thematic classification of stranger rapes. Behavioral Sciences and the Law, 21, 157-174.

CANTeR, D. V., y Heritage, R. (1990). A multivariate model of sexual offence behaviour: Developments in 'offender profiling'. The Journal of Forensic Psychiatry, 1(2), 185-212.

CANTER, D. V., y Youngs, D. (2009). Investigative psychology: Offender profiling and the analysis of criminal action. West Sussex, UK: Wiley.

Chen, H., Cohen, P., y Chen, S. (2010). How big is a big odds ratio? Interpreting the magnitudes of odds ratios in epidemiological studies. 
Communications in Statistics - Simulation and Computation, 39, 860864.

Corovic, J., Christianson, S. A., y Bergman, L. R. (2012). From crime scene actions in stranger rape to prediction of rapist type: singlevictim or serial rapist? Behavioral Science and the Law, 30, 764-781.

Douglas, J. E., Ressler, R. K., Burgess, A. W., y Hartman. C. R. (1986). Criminal profiling from crime scene analysis. Behavioral Sciences $y$ the Law, 4, 401-421.

GibBens, T. C., Way, C., y SoothiLl, K. L. (1977). Behavioural Types of Rape. British Journal of Pyschiatry, 130, 32-42.

Goodwill, A. M., Alison, L. J., y BeEch, A. R. (2009). What works in offender profiling? A comparison of typological, thematic, and multivariate models. Behavioral Sciences y the Law, 27(4), 507-529.

Goodwill, A. M., Lehmann, R. J. B., Beauregard, E. y Andrei, A. (2016). An action phase approach to offender profiling. Leg Crim Psychol, 21: 229-250.

GreEnfeld, L. A. (1997). Sex Offenses and Offenders. An Analysis of Data on Rape and Sexual Assault. U.S. Department of Justice. Bureau of Justice Statistics.

HäKKänen, H., LindlöF, P., y SAnttila, P. (2004). Crime scene actions and offender characteristics in a sample of Finnish stranger rapes. Journal of Investigative Psychology and Offender Profiling, 1, 17-32.

Hewitt, A.N., Chopin, J., y Beauregard, E. (2020) Offender and victim 'journey-to-crime': Motivational differences among stranger rapists. Journal of Criminal Justice, Volume 69.

Holmstrom, L. L., y Burgess, A. W. (1980). Sexual behavior during reported rapes. Archives of Sexual Behavior, 9, 427-446.

JANOSCH GonzÁLez, H. (2020) La perfilación criminológica desde la perspectiva de los problemas inversos según el enfoque de Mario Bunge, en El resurgimiento de la Criminología científica en América Latina. Estudios en homenaje al Profesor Ayar Chaparro Guerra con motivo de su $75^{\circ}$ cumpleaños (11.4.2020) (Alfonso Serrano Maíllo y José Luis Guzmán Dalbora eds.), Dykinson, Madrid.

Lehmann, R. J. B., Goodwill, A. M., Gallasch-Nemitz, F., Biedermann, J., y DAHLE, K.-P. (2013). Applying crime scene analysis to the prediction of sexual recidivism in stranger rapes. Law and Human Behavior, 37(4), 241-254.

Mokros, A., y Alison, L. J. (2002). Is offender profiling possible? Testing the predicted homology of crime scene actions and background characteristics in a sample of rapists. Legal and Criminological Psychology, 7(1), 25-43. 
Pérez Ramírez, M., Sánchez LóPez, M., Giménez-Salinas Framis, A., y de Juan Espinosa, M. (2018). Stranger rape: classifying Spanish sexual offences using multiple correspondence and cluster analyses. Journal of Sexual Aggression, Vol. 24, 2, 225-239.

Pueyo, A. A. y Redondo Illescas, S. (2007). Predicción de la violencia. Entre la peligrosidad y la valoración del riesgo de violencia. Papeles del Psicólogo, 28(3), 157-173.

Secretaría de Estado de Seguridad del Ministerio del Interior (2018). Informe sobre Delitos contra la Libertad e indemnidad Sexual en España.

Snook, B., Eastwood, J., Gendreau, P., Goggin, C., y Cullen, R. M. (2007). Taking Stock of Criminal Profiling: A narrative Review and Meta-Analysis. Criminal Justice and Behavior, 34, 437-453.

Soto Castro, J. E. (2017). Manual de investigación psicológica del delito, $2^{\mathrm{a}}$ edición. Madrid, España: Pirámide.

Sotoca Plaza, A., González Álvarez, J. L., y Halty Barrutieta, L. (2019) Perfiles criminales. Principios, técnicas y aplicaciones. Síntesis: Madrid.

Turvey, B.E. (Ed.) (2012). Criminal profiling: An introduction to behavioral evidence analysis. London, UK: Elsevier. 